\title{
THE IMPACT OF POLARIMETRY ON WOLF-RAYET STAR MODELS
}

\author{
REGINA E. SCHULTE-LADBECK \\ Space Astronomy Laboratory, University of Wisconsin - Madison \\ 1150 University Ave., Madison, WI 53706, U.S.A.
}

Recent observational efforts have presented new results concerning (linear) polarization variations as a function of time as well as wavelength that have considerable impact on WR star models:

Polarimetry throughout orbital phase has provided inclinations of double-line spectroscopic binaries containing WR stars with $\mathrm{O}$ star companions, thus improving our understanding of the masses and evolution of WR binaries (Schulte-Ladbeck 1989, A.J. 97, 1471). New binary-model calculations were presented at the meeting and continuing comparison with observations should prove productive.

Temporal polarization variations in single WR stars show various types of behavior: none, stochastic, along a preferred axis in the Stokes' parameter plane (see SchulteLadbeck and van der Hucht 1989, Ap.J. 337, 872 and references therein). This is indicative of a variety of atmospheric properties, i.e. occurrence of non-spherical geometries and non-steady dynamics in WR star winds (perhaps related to WR subtype?). Polarimetry demonstrates that spherically symmetric, purely radiatively-driven winds do not describe all WR stars. In a growing number of stars, polarimetry implies random "blob" ejections in the winds, while for a few stars a case can be made in favor of rotation. More such observations are clearly needed for the single WR stars.

At the Space Astronomy Laboratory, our interest has focussed on studying the winds of WR stars through spectropolarimetry. Line-polarization features expected from stratification of ionization and excitation in an extended, asymmetric atmosphere have only been detected in three WN5-6 stars but, curiously, in none of the observed WC stars (see Schmidt 1988 in "Polarized Radiation of Circumstellar Origin", p.641). Observations from our Pine Bluff Observatory's 36" telescope covering $3200 \AA$ to $7800 \AA$ at a resolution of $\sim 30 \AA$ confirm the depolarization across emission lines in EZ CMa (WN5). We have obtained a spectropolarimetric data set covering various phases of the star's 3.766-day period which shows that both the continuum and line polarization are variable. The line effect is most pronounced in the strong line of He II at $4686 \AA$, and we find that the polarization changes across the line profile. In the QU plane, the polarization line profile traces a loop. Such behavior has previously been observed in the Balmer lines of Be stars, where it has been modeled through the line absorption in an electron scattering, expanding, rotating and inclined disk. A WR star not previously studied with spectropolarimetry is HD 193793 (WC7+O4-5). We find the polarization spectrum to be smooth and featureless (S/N of about 20 in the polarization); it can be explained as caused primarily by interstellar polarization.

Our optical spectropolarimetric observations of WR stars will soon be extended into the ultraviolet spectral range, thus giving access to different line transitions. Polarization measurements ranging from $1400 \AA$ to $3300 \AA$ at a resolution of $\sim 6 \AA$ are scheduled to be obtained with the Wisconsin Ultraviolet Photo-Polarimeter Experiment (WUPPE). This 20" telescope is part of the Astro-1 space-shuttle mission currently scheduled to fly in mid August, 1990.

It is a pleasure to thank the PBO reducers team. This work is supported by NASA contract no. NAS5-26777. 